\title{
Histoire de la philosophie médiévale
}

Alain de Libera

\section{OpenEdition}

Journals

Édition électronique

URL : https://journals.openedition.org/annuaire-cdf/15819

DOI : 10.4000/annuaire-cdf.15819

ISBN : 978-2-7226-0572-5

ISSN : 2109-9227

Éditeur

Collège de France

Édition imprimée

Date de publication : 30 décembre 2020

Pagination : 379-393

ISBN : 978-2-7226-0516-9

ISSN : 0069-5580

Référence électronique

Alain de Libera, « Histoire de la philosophie médiévale », L'annuaire du Collège de France [En ligne], 118 2020, mis en ligne le 01 avril 2021, consulté le 22 août 2022. URL : http://journals.openedition.org/ annuaire-cdf/15819; DOI : https://doi.org/10.4000/annuaire-cdf.15819 


\title{
HISTOIRE DE LA PHILOSOPHIE MÉDIÉVALE
}

\author{
Alain DE LIBERA \\ Membre correspondant de l'Académie des inscriptions et belles-lettres, \\ professeur au Collège de France
}

Mots-clés : philosophie médiévale, Heidegger, Foucault, existence, vérité

La série de cours "Destructionis destructio (II). Existence et vérité » est disponible, en audio et vidéo, sur le site internet du Collège de France (https://www.college-de-france. $\mathrm{fr} / \mathrm{site} /$ alain-de-libera/course-2017-2018.htm), ainsi que le colloque « L'Europe de la logique » (https://www.college-de-france.fr/site/alain-de-libera/symposium-2017-2018. htm). La journée d'études « Gilson \& Blumenberg » (https://www.college-de-france.fr/ site/alain-de-libera/studyday-2017-2018.htm) est quant à elle disponible sur le site en version audio.

\section{ENSEIGNEMENT}

\section{COURS - DESTRUCTIONIS DESTRUCTIO (II). EXISTENCE ET VÉRITÉ}

Donné sur quatorze leçons d'une heure, le cours a poursuivi la confrontation entre histoire de l'Être (Sein) et/ou de l'Estre (Seyn) selon Heidegger et histoire de la vérité selon Foucault en examinant d'un point de vue archéologique leurs restitutions respectives de la généalogie du rapport entre existence et vérité, à partir de la «victoire » alléguée de la «philosophie » platonico-aristotélicienne et de l'« apophantique » sur la «sophistique » - restitutions toutes deux caractérisées par des formes diverses de méconnaissance ou de sous-évaluation de la pensée médiévale, qu'elle soit philosophique ou théologique. Partant de la critique d'Aristote dans les Leçons sur la volonté de savoir, on a, en outre, trouvé chez le Foucault du Courage de la vérité une prise de distance significative à l'égard des thèses de Heidegger sur le nihilisme, imposant une relecture critique du thème du «philosophe essentiel ».

\section{5 février 2018}

Dans la première des Leçons sur la volonté de savoir (9 décembre 1970), Foucault se donnait comme objectif l'histoire d'une « double transformation » : (1) la naissance 
$\mathrm{du}$ « discours philosophico-scientifique », autrement dit, de la philosophie ; et (2) les rendant possibles : la «liquidation» de la «sophistique» au profit de l'«apophantique ». Ce qui se mettait ainsi en place en 1970/1971, dans le premier cours du Collège de France, n'annonçait en rien les publications sur l'histoire de la sexualité, engagées en 1976 avec La Volonté de savoir, pour s'achever en janvier 2018 avec Les Aveux de la chair. Il y allait, en effet, principalement d'une critique d'Aristote. L'expression « volonté de savoir » parallèle à la « volonté de puissance » imposant à la fois une référence directe à Nietzsche et, indirecte, à l'interprétation heideggérienne de Nietzsche, le cours de Foucault nouait deux fils : l'un aristotélicien, l'autre heideggérien. Poursuivant la déconstruction du «Grand récit » heideggérien, nous avons, en 2018, principalement suivi le premier, en nous concentrant sur l'idée foucaldienne de «partage violent du vrai et du faux », l'un des éléments du « système d'exclusion » censé régir la volonté de savoir, «en tant qu'elle exerce elle-même un rôle d'exclusion sur le discours ». Il y a, de fait, pour Foucault un événement dans l'histoire de la vérité qui coïncide avec la naissance de la philosophie, la rend possible, et lui donne forme : le «déplacement de la vérité », qui s'opère dans le monde grec entre le $\mathrm{VI}^{\mathrm{e}}$ et le $\mathrm{V}^{\mathrm{e}}$ siècle avant J.-C. Après un rappel des emprunts de Foucault aux «analystes anglais », Austin et Searle, permettant d'articuler la distinction de L'Archéologie du savoir entre phrases, propositions, énoncés et speech acts, on a évoqué l'analyse de ce «déplacement de la vérité », présenté dans le cours de 1970 comme le déplacement opéré par Platon de l'acte d'énonciation vers l'énoncé luimême, puis, presque au bout du parcours, en 1982-1983, dans Le Gouvernement de soi et des autres, l'analyse de la «dramatique du discours vrai », définie comme celle des «faits de discours qui montrent comment l'événement même de l'énonciation peut affecter l'être de l'énonciateur» qui a le courage de dire vrai. On a ainsi été amené à distinguer les thèses platoniciennes de 1983 sur le « réel de la philosophie » tirées de la relecture foucaldienne de la Lettre VII, requérant de la philosophie et du

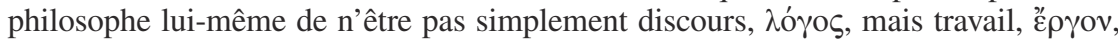
plein acte de véridiction, et les thèses platonico-aristotéliciennes sur le logos, analysées en 1970-1971, comme fondant la « logique».

Dans les leçons des 6 et 13 janvier 1971, Foucault évoque les deux opérations distinctes, mais complémentaires, opérées par Platon avec la liquidation des sophistes, puis Aristote, avec la pérennisation des sophismes. C'est dans l'analyse de cette pérennisation qu'il glisse quelques remarques sur le Moyen Âge. Cette analyse répète elle-même le geste d'exclusion de la logique médiévale opéré par Kant, affirmant que depuis Aristote la logique «n'avait pu faire un seul pas en avant». Concernant l'événement du « déplacement de la vérité », on a montré que Foucault déplaçait luimême sur le personnage du sophiste un élément central de l'analyse de la fonction du poète dans l'œuvre qui porte en sous-main ses analyses - le livre «jamais cité » de Marcel Detienne, Les Maîtres de vérité dans la Grèce archä̈que, paru chez Maspero en 1967 : le fait d'exercer un pouvoir, celui de « dire la vérité ». Après avoir évoqué

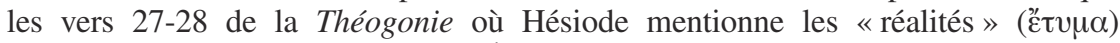
factuelles, puis les « vérités » $(\dot{\alpha} \lambda \eta \theta \dot{\varepsilon} \alpha)$ proférées, on a repris en détail les éléments de la vérité $(\dot{\alpha} \lambda \eta \dot{\eta} \theta \varepsilon \iota \alpha)$ poétique selon Detienne: une vérité «assertorique», « fondamentalement différente de notre conception traditionnelle »; une vérité qui ne consiste ni dans «l'accord de la proposition et de son objet», ni dans celui «d'un jugement avec les autres jugements », car, dans la Grèce archaïque, l'opposé de la vérité n'est pas le «faux », mais l'oubli $(\Lambda \eta \dot{\theta} \theta \eta)$. On a abordé ensuite la critique de Detienne par Bernard Williams, puis commencé, sur cette base, l'examen de la 
critique de Foucault par Jacques Bouveresse. On s'est arrêté à ce propos sur la notion d'alèthurgie, introduite dans le cours de 1979-1980 sur le gouvernement des vivants comme «l'ensemble des procédés possibles, verbaux et non verbaux, par lesquels on amène à jour ce qui est posé comme vrai, par opposition au faux, au caché, à l'indicible, à l'imprévisible, à l'oubli », puis, ayant rappelé le principe foucaldien stipulant qu'il n'y a «pas d'exercice du pouvoir sans une alèthurgie », on a présenté deux critiques de Bouveresse : 1) Foucault ne prend pas en considération l'usage ordinaire du langage ; 2) il ne considère jamais « la question de la vérité a parte rei, mais toujours uniquement a parte veridictionis et plus précisément a parte veridicentis ». Cette dernière formule a été l'occasion de revenir sur la distinction entre "véridicteur », " vérifacteur» (truthmaker) et "vériporteur» (truthbearer), puis de souligner le rôle de la «véridiction» dans la sémiotique du discours et la sémantique textuelle d'A.J. Greimas, parties intégrantes du champ de présence de Foucault, au moment où celui-ci met en place son travail sur le dire-vrai et la parrêsia.

\section{2 février 2018}

Selon J. Bouveresse, la définition foucaldienne de l'histoire critique de la pensée par l'« émergence des jeux de vérité » neutralise la distinction frégéenne entre êtrevrai et être-tenu pour vrai, en ramenant le premier au second. Après avoir rappelé les thèses de Frege sur le troisième domaine, on s'est concentré sur la notion foucaldienne d'alèthurgie comme manifestation/production de la vérité, et sur l'ambiguïté du terme "produire » (une chose/un témoignage). On a évoqué ensuite les thèses des conférences de Rio de Janeiro sur La Vérité et les formes juridiques (mai 1973) : passage de l'épreuve judiciaire (médiévale) à l'enquête, distinction entre « opérateur de droit » et « opérateur de vérité » (apophantique), et souligné que la question du sujet de l'action en tant que Wer-Frage (question QUI ?) prolongeait l'opération apophantique d'Aristote dans l'ordre judiciaire. De là, on s'est intéressé à l'opposition entre relativisme foucaldien et réalisme aristotélicien brossée par Bouveresse. La thèse de Foucault sur la vérité (notée ici TF/TF*) s'analyse en TF : ce n'est pas parce que le dit du dire-vrai est vrai que le dire-vrai peut être dit « vrai »; c'est parce que le dire-vrai est vrai que son dit peut être dit «vrai »; et TF* : la vérité n'est pas la cause, mais l'effet de la connaissance. Selon Bouveresse, TF s'oppose directement à Aristote, Métaphysique, $\Theta$ 10, 1051b7-10 («Ce n'est pas parce que nous pensons d'une manière vraie que tu es blanc, que tu es blanc, mais c'est parce que tu es blanc, qu'en disant que tu l'es, nous sommes dans la vérité. »). On peut faire la même critique en s'appuyant sur $\Gamma$ 7, 1011b 26. C'est ce que fait Bouveresse en esquissant une comparaison entre Foucault et Hacking. On doit toutefois noter que beaucoup de choses dépendent ici des traductions. Comme on l'a montré, les traductions de Kirwan et de Ross utilisées par Hacking ne disent pas la même chose que celle de Tricot, ni que celle de B. Cassin et M. Narcy dans $L a$ Décision du sens, traduction et commentaire de Métaphysique, $\Gamma^{1}$. Selon Hacking Aristote et Austin se rejoignent sur deux points : «Ils parlent de ce qu'on dit, et ils parlent de l'adjectif "vrai” ». Pour Bouveresse, ce n'est pas le cas de Foucault: celui-ci parle «la plupart du temps de l'expression "dire-vrai", considérée comme

1. Barbara CASSIN et Michel NARCY, La Décision du sens. Le livre Gamma de la Métaphysique d'Aristote, Paris, Vrin, 1989. 
un tout et dans laquelle "vrai" ne fonctionne pas vraiment comme un adjectif, mais plutôt comme une sorte d'adverbe ». On a noté que, en ces termes, Foucault semblait soutenir une théorie adverbiale de la vérité du type de celles présentées dans notre séminaire de 2015 sur les fonctions psychiques. On a conclu en soulignant que la confusion entre l'être-vrai et le tenir-pour-vrai imputée à Foucault via Frege ouvrait sur la problématique médiévale de l'assentiment (assensus).

La deuxième heure a permis d'aborder le bannissement du sophiste. Selon Foucault (leçon du 6 janvier 1971), le principal acteur n'en est pas Platon, mais Aristote. Platon a «liquidé » les sophistes, leur philosophie et leur « règle de vie » purement « chrématistique », tournée vers le succès et le gain. Aristote a préservé les sophismes, qu'il a inclus pour mieux les maîtriser. Foucault dit peu de choses du destin des sophismes après Aristote. Il évoque en quelques lignes les insolubilia et les sophismata médiévaux, en s'appuyant principalement sur The Development of Logic de William et Martha Kneale (1962). Il ignore les travaux d'histoire de la logique médiévale, qui prennent pourtant exactement leur essor aux confins des années 1960-1970, aux Pays-Bas (L.M. De Rijk), au Danemark (J. Pinborg, S. Ebbesen), aux États-Unis (N. Kretzmann) et en Italie (A. Maierù). Il s'intéresse exclusivement à l'opposition entre opération apophantique et opération sophistique ou «éristique» (du grec ěpıৎ, «dispute»). Le cours du 13 janvier 1971 définit l'opération apophantique comme le « geste sans cesse renouvelé par lequel le rapport d'un énoncé à la réalité, à l'être, à la vérité est dénoué au niveau de l'événement énonciatif et reporté à ce qui est dit dans l'énoncé et au rapport entre ce qui est dit et les choses elles-mêmes ». Cette formule est une version étendue de l'événement intervenu «entre Hésiode et Platon»: le déplacement de la vérité de l'acte d'énonciation à l'énoncé lui-même. Les pages que Foucault consacre à la liquidation en deux temps du sophiste et de la sophistique montrent qu'il a une conscience claire des réquisits de ce que Bouveresse appelle le «réalisme». La domination qui s'exerce sur le personnage du sophiste dans le dialogue éponyme de Platon a un double point d'appui : 1) «l'affirmation qu'on accède à la vérité dans une discussion que l'on mène avec soi-même dans son propre esprit »;2) l'affirmation, liée à la précédente, que « dire faux, c'est dire que ce qui est n'est pas » (Sophiste 263D). Ce sont ces deux affirmations que l'on retrouve déplacées et intériorisées chez Aristote, dans la Métaphysique, $\Gamma 7$, «quand il définit l'énoncé vrai par le fait de dire que ce qui est est et que ce qui n'est pas n'est pas », et dans les Seconds Analytiques, " quand il dit que le syllogisme et la démonstration n'ont pas affaire au discours extérieur mais à celui qui se tient dans l'âme » (I, 10, 76b24-27). On a conclu la séance en pointant les quatre phénomènes ayant selon Foucault « donné fondement à la science et à la philosophie occidentales dans leur développement historique »: 1) «l'exclusion de la matérialité du discours »,2) « l'émergence d'une apophantique donnant les conditions auxquelles une proposition peut être vraie ou fausse », 3) « la souveraineté du rapport signifiant/signifié », 4) «le privilège accordé à la pensée comme lieu d'apparition de la vérité ».

\section{6 février 2018}

La thèse de Foucault faisant de l'apophansis aristotélicienne ce qui «donne fondement » au discours philosophico-scientifique peut être présentée largo sensu comme heideggérienne. Partant de la conférence de Montréal sur Nietzsche d'avril 1971, on a cependant suivi chez Foucault l'émergence d'un modèle 
« nietzschéen » de l'invention de la connaissance et de la vérité distinguant, comme dans les LVS, origine (Ursprung) et invention (Erfindung). Le rejet foucaldien de l'origine est un rejet de la naturalité, un rejet de la nature humaine, donc du désir naturel de connaître invoqué par Aristote. Selon D. Defert, en opposant dans la conférence de Montréal le paradigme d'Aristote à un paradigme nietzschéen, Foucault s'oppose à l'interprétation heideggérienne de l'histoire de la philosophie. 1) Il « réinscrit l' "Ouverture" heideggérienne dans l'histoire de la métaphysique inaugurée par Platon », en réponse aux deux volumes de Heidegger sur Nietzsche, qui prétendent inscrire Nietzsche «dans la tradition métaphysique qu'il a voulu subvertir». 2) Il termine sa conférence par une violente diatribe contre «l'idéologie du savoir comme effet de la liberté », où il est difficile de ne pas reconnaître les thèses de Heidegger sur la liberté comme essence de la vérité, dans le chapitre 4 de Vom Wesen der Wahrheit, présentant «l'apérité du comportement 》 (qui rend possible la "conformité » entre pensée et choses), comme "fondée dans la liberté ». Après une enquête sur les notions d'Offenheit (ouverture, apérité) et de Verhalten (comportement) chez Heidegger, et une reprise des thèses de Vom Wesen der Wahrheit sur l'Ouvert comme

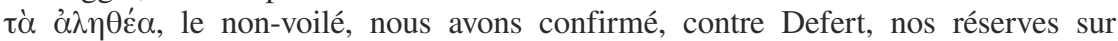
l'utilisation du Nietzsche de Heidegger par le Foucault des LVS, mais souscrit, en revanche, à son interprétation du «contournement critique» de Heidegger par Foucault, fondée sur l'idée d'un déplacement du «partage heideggérien de la philosophie », opéré en s'appuyant sur Hésiode plutôt que sur Héraclite, sur les sophistes plutôt que sur les présocratiques, sur l'histoire plutôt que la philologie, et surtout sur un rejet de l' «idéologie du savoir comme effet de liberté ». Cela posé, nous avons insisté sur ce que Foucault et Heidegger avaient en commun : rien de moins que l'alêthêia et le logos apophantikos. C'est cela seul qui importe pour penser leur relation respective au Moyen Âge ; cela seul aussi qui compte du point de vue du champ de présence foucaldien. Si l'on peut hésiter sur les termes définissant la relation de Foucault à Heidegger («contournement critique», «confrontation», « contre-récit»), c'est en effet chez Heidegger que Foucault peut trouver de quoi problématiser sur sa ligne de faîte le rapport entre alêthêia et lêthê. De même, c'est chez lui que figure le texte canonique ouvrant si l'on ose dire la possibilité d'une interprétation authentiquement «apophantique d du logos apophantikos: le § 7, section B, de Sein und Zeit, traduit dès 1964 par R. Boehm et A. de Waelhens.

Heidegger rejette l'interprétation moderne, néokantienne (Rickert, Windelband) et postbrentanienne du $\lambda$ ó $\gamma$ os comme jugement, qu'il s'agisse du jugement comme liaison ou synthèse (Verbinden), ou comme prise de position (Stellungnahme): acquiescement (Anerkennen) ou refus (Verwerfen). Dès ses premiers travaux, il critique les théories psychologistes du jugement de Wundt, Maier, Brentano, Marty

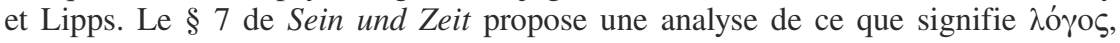
marquée par le souci de revenir au véritable sens de la notion de logos apophantikos chez Aristote. Le paragraphe est articulé sur deux grandes thèses : la première est que « le logos est un faire-voir » (Sehenlassen), la seconde que « le logos n'est pas le lieu primaire de la vérité ». On a examiné les deux, en renvoyant ici ou là aux analyses préparatoires du cours du semestre d'hiver 1925-1926, Logik. Die Frage nach der Wahrheit (Logique : la question de la vérité), sur la fonction " délotique »

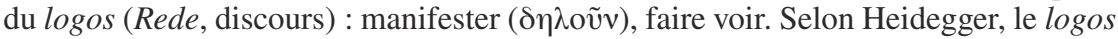
comme apophansis a une structure synthétique : il fait voir quelque chose (etwas) avec quelque chose (mit etwas), comme quelque chose (als etwas) - ce pourquoi il peut être vrai ou faux. La thèse dite de la «vérité correspondance », de la vérité 
comme « accord », ou « conformité » (formulée par Tarski en référence à $\Gamma$ 7, 1011b 26 et alléguée par Bouveresse contre Foucault), n'est pas aristotélicienne. «L'êtrevrai du $\lambda$ ó $\gamma \circ \varsigma$ comme $\alpha \lambda \eta \theta \varepsilon v ́ \varepsilon ı v$ veut dire soustraire à son retrait, dans le $\lambda \varepsilon^{\prime} \gamma \varepsilon ı v$

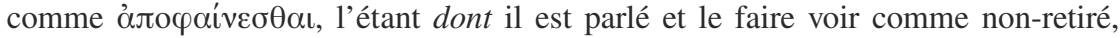
$(\hat{\alpha} \lambda \eta \theta \dot{\varepsilon} \varsigma)$ le découvrir : Ent-decken ». Le $\S 11$ du cours de 1925-1926 dénonce trois erreurs traditionnelles sur la vérité : T1 : le lieu de la vérité est la proposition; T2 : la vérité est l'accord de la pensée avec l'étant; T3 : ces deux énoncés trouvent leur origine chez Aristote. Pour établir le caractère erroné de l'interprétation traditionnelle, le $\S 7$ de $S u Z$ pose deux questions, auxquelles il apporte réponse. On les a examinées en détail. La première question est Q1 : Qu'est-ce qui est vrai au sens grec? S'appuyant sur Aristote, Heidegger répond (R1): ce qui est vrai premièrement ce sont les sens. Or les sens ne «jugent» pas. Est donc « vraie » au

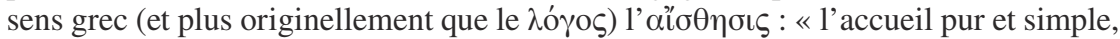
sensible de quelque chose ». La deuxième question est Q2 : Qu'est-ce qui au sens grec est vrai au sens le plus pur et le plus originel ? La réponse de Heidegger, tirée d'Aristote, est R2 : « au sens le plus pur est vrai le noein (voeiv), l'accueil purement et simplement considératif des déterminations d'être les plus simples de l'étant comme tel ». Les textes allégués en faveur de R1 et R2 sont deux passages du De anima : De an. 427 b 11 sq. pour R1 ; De an. 430a26 sq. pour R2.

\section{5 mars 2018}

La première heure du cours a été consacrée à l'analyse de R1 et R2. On a d'abord considéré les textes supportant R1 (De anima, III, 3, 427b11-14 ; II, 6, 418 a 7-25 ; II, 6, 418a20-25; III, 3, 428b18-22), et expliqué en quoi, tout en ne « jugeant » pas, les sens sont toujours dans le vrai quant à leur sensible propre : l'ouïe " découvre » toujours du son, la vue, de la couleur, le goût, une saveur. On a ensuite examiné R2, et sa source : 430a26. Il s'agit du passage du De anima sur "l'intellection des

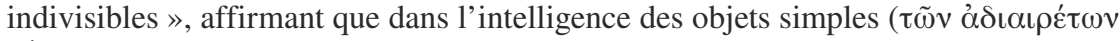

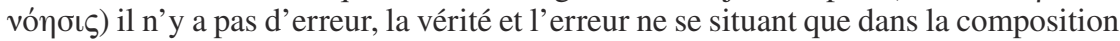
des notions ( $\sigma u ́ v \theta \varepsilon \sigma i \zeta \zeta v o \eta \mu \alpha ́ \tau \omega v)$. On a suivi l'élaboration de cette thèse au Moyen Âge, en analysant la théorie de la «triple opération de l'intellect»: O1: l'appréhension de la quiddité (quod quid est) ou essence (essentia) en elle-même ; $\mathrm{O} 2$ : l'opération de l'intellect qui compose et divise ; O3 : le «raisonnement» (operatio ratiocinandi), où la raison "procède du connu à l'inconnu » (de notis ad ignota). Ces formules, empruntées à Siger de Brabant et Thomas d'Aquin nous ont permis de mettre une nouvelle fois en évidence l'influence d'Avicenne. La distinction entre quid est et esse vel non esse renvoie, en effet, à la distinction avicennienne d'essence et d'existence; la description du raisonnement comme procédant du connu à l'inconnu à la Logica Avicennae, traduction latine de l'Isagoge du Šifā' par Avendauth (éditée critiquement par F. Hudry en 2018). L'élévation par Albert le Grand de l'inférence cognitive de notis ad ignota au rang de «méthode commune à toute science » témoigne d'une volonté de savoir propre au Moyen Âge, illustrant, au passage, le bien-fondé du schéma narratif de la translatio studiorum, avec ses deux filières de traduction/transmission : gréco-latine et arabo-judéo-latine. L'Isagoge du Šifä' prolonge l'enseignement des Introductions à la philosophie

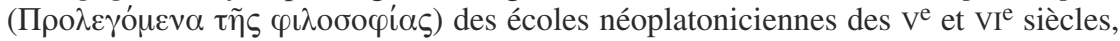
comme le font les textes de Gundissalinus et d'Alfārābī sur la «division » des sciences, qui souvent l'accompagnent. Les textes «didascaliques» des années 
charnières du XIII ${ }^{\mathrm{e}}$ siècle où se met en place la "philosophie universitaire », qui exploite à fond la connexion entre logique et psychologie, héritent à travers ces textes de ce qu'on pourrait appeler "l'univers d'Ammonius", avant même la traduction de son Commentaire sur le Perihermeneias par Guillaume de Moerbecke en 1268 et son utilisation par Thomas d'Aquin dans son Expositio, au début des années 1270. La distinction des trois opérations de l'intellect sert aux scolastiques à établir l'ordo de la discipline logique. À la formation des concepts simples correspondent les Catégories, aux jugements, le Perihermeneias, aux raisonnements, les Analytiques, les Topiques et les Réfutations sophistiques.

La seconde partie de la leçon a permis de reprendre l'examen de R2. Heidegger affirme que, pour Aristote, le « pur vовĩv » ne peut jamais « recouvrir », qu'il peut

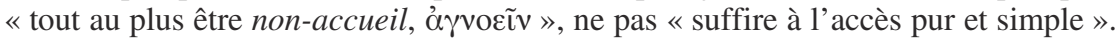

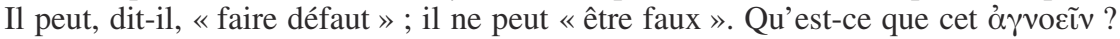
Ce n'est pas dit en De anima, 430a26, mais en Métaphysique, $\Theta 10$, un des textes sur lesquels J. Bouveresse $\mathrm{a}$, en l'espèce de $1051 \mathrm{~b} 7-10$, fondé sa critique de la réduction foucaldienne de l'être-vrai à la véridiction. On a analysé ce texte, puis on est revenu à Heidegger. Le cœur de l'interprétation heideggérienne du «pur voعĩv » dans le $\S 7$ de $S u Z$ est l'articulation entre le « délotique » et «l'apophantique », qui commande la thèse sur l'origine du faux. Ce qu'on appelle la « vérité du jugement » est seconde par rapport à la structure synthétique du faire-voir apophantique. En fait, il y a deux sortes d'Ent-decken (découvrir) : l'un qui ne comporte pas, l'autre qui comporte la possibilité du Ver-decken (recouvrir). Le $\delta\rceil \lambda o \tilde{v}$ (découvrement) qui ne peut recouvrir est le «pur voeĩv»; l'autre est le logos apophantique, l'aufweisende Sehenlassen, qui peut être découvrant ou occultant. La structure synthétique du découvrement apo-phantique autorise les deux. Le découvrir qui "fait-voir » une chose $=x$ (etwas) en recourant à autre chose $=y$ (mit etwas) ouvre ce faisant la possibilité de recouvrir $x$, de placer $y$ devant $x$ et de faire voir $x$ comme ce qu'il n'est pas. Il ouvre la possibilité du faux. La « vérité du jugement» n'est que «le cas opposé du recouvrement». Faute de temps, il n'a pas été possible de compléter l'analyse du § 7 de SuZ par les textes du cours de 1924-1925 sur le Sophiste (GA 19, $\S 80$ : 601), où Heidegger caractérise l'etwas als etwas, le «caractère-d'en-tantque » (Als-Charakter) comme la « catégorie logique proprement dite » (die eigentlich logische Kategorie), ni par ceux du cours de 1925-1926, où il souligne que la

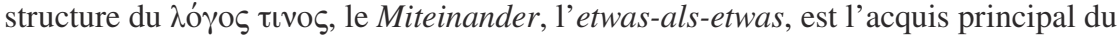
Sophiste (GA $21: 142$ : Die Rede ist Rede über etwas und von etwas).

\section{2 mars 2018}

La première heure a été consacrée à un résumé détaillé des thèses de $S u Z$ § 7 sur la structure phénoménale de ce que le cours sur le Sophiste appelle l'Ansprechbarkeit (l'«advocabilité »), i.e. l'appréhensibilité du « quelque chose » en tant que « quelque chose ». Le nom de la structure synthétique qui, faisant voir l'être-ensemble de $x$ et de $y$, ouvre chez Aristote la possibilité de « recouvrir » $x$ et par là-même ouvre celle du

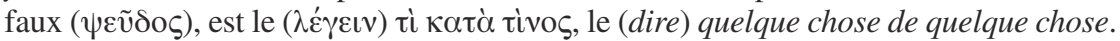
L'expression de «structure » étant employée par Ernst Tugendhat, dans Tí kata tinós. Eine Untersuchung zu Struktur und Ursprung aristotelischer Grundbegriffe (1958), on a examiné ses thèses et les commentaires de P. Aubenque dans sa recension. Après

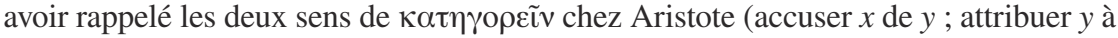
$x$ ), on est passé à l'analyse du De anima, III, 6, 430b26-31. Le texte a été examiné 
ligne par ligne en confrontant les traductions françaises de Tricot, Barbotin, Thillet et Bodéüs avec les traductions latines de Jacques de Venise (translatio vetus du De anima, sur le grec) et de Michel Scot (translatio nova, accompagnant la traduction du Grand Commentaire d'Averroès). L'analyse du textus 26 du livre III du De anima et du commentaire correspondant d'Averroès a été l'occasion de s'arrêter sur la distinction entre la «représentation» ou «conception» (formatio intellectiva, informatio, ar. tașawwur) et l' «assentiment » (fides, ar. tașdīq), introduite dans le commentaire 21, comme « la plus notoire (famosior) des différences qui caractérisent l'action de l'intellect ». On en a proposé une reconstruction archéologique en partant de la Logica Avicennae, traduite par Avendauth, distinguant deux modes de connaissance: selon l'intellect (secundum intellectum), c'est-à-dire ici la représentation, et selon la «croyance » - littéralement la «crédulité » (secundum credulitatem), c'est-à-dire l'assentiment. On a suivi ce duo chez Gundissalinus, avec la distinction entre imaginatio et credulitas, chez Ghazālī, dans le Maqāṣid al-falāsifa (traduit par Avendauth et Gundissalinus sous le titre De intentionibus philosophorum), chez Albert le Grand, avec la distinction entre formatio per intellectum (conception) et fides (assentiment). On a conclu ce parcours en examinant la formulation de la distinction entre science et opinion chez Buridan, où l'on retrouve non seulement le tașdī $q$ sous le vocable latin d'assensus, mais encore la notion épistémique de « crainte de l'opposé », clairement héritée d'Avicenne (« scientia est assensus firmus et opinio est assensus cum formidine ad oppositum »). On a achevé la lecture du $§ 7$ de SuZ en soulignant que, lisant le Perihermeneias, Heidegger interprétait l'élément dit « grec » de la pensée d'Aristote en faisant intervenir un autre texte : le De anima. Ce fait nous a conduit à examiner ce qui dans l'interprétation heideggérienne du logos apophantikos, comme dans le dossier foucaldien de «l'opération apophantique », relevait archéologiquement de l'histoire des corpus, élément central de que nous appelons avec Granel traditionis traditio.

Le lien entre le Perihermeneias et le De anima est fait par Aristote lui-même. Le premier chapitre du Perihermeneias introduit une structure articulant les mots parlés ( $\varphi \omega v \alpha i)$, les états de l'âme ( $\pi \alpha \theta \dot{\eta} \mu \alpha \tau \alpha \tau \tilde{\eta} \varsigma \psi v \chi \tilde{\eta} \varsigma$, passiones animae), et les choses $(\pi \rho \alpha ́ \gamma \mu \alpha \tau \alpha)$ : le «triangle sémantique d'Aristote». Dans le Perihermeneias, Aristote ne développe pas son dispositif en s'interrogeant sur le type de rapport existant entre mots, concepts et choses - il renvoie au De anima. « Tout cela, dit-il, a été traité dans notre livre de l'Âme, car cela intéresse une discipline différente »:

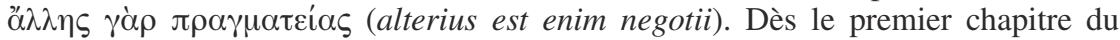
Perihermeneias, la distinction entre le logique et le psychique - ce qui portera l'opposition structurante pour le jeune Heidegger entre logicisme et psychologisme - est apparue comme programmée. On est donc revenu sur la question du « lieu de la vérité ». Quel est le lieu du vrai selon le Perihermeneias ? La réponse est double : l'âme et la voix. Elle est donnée en 16a9-13. Il en va de même dans l'âme ( $\psi v \chi \tilde{\eta})$ et dans la voix ( $\dot{\varepsilon} v \tau \tilde{\eta} \varphi \omega v \tilde{\eta})$ : le vrai et le faux consistent dans une composition et une division. Le «verbe» est ce qui assure le bouclage de la «structure » du legein ti kata tinos. Analysant le passage définissant ce qu'est un verbe nous avons montré que, dans sa fine pointe (16b9-10), il avait été depuis Boèce

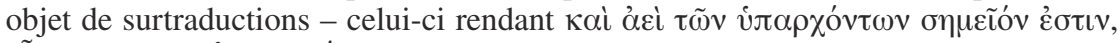

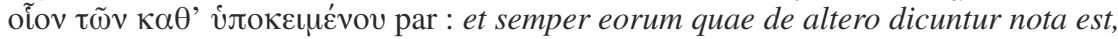
ut eorum quae de subiecto vel in subiecto. En bref, les traductions latine et modernes (française, anglaise, allemande) introduisent dans la définition du verbe, censée ouvrir l'exposé des rudiments de sémantique nécessaires à la mise en place de la 
forme propositionnelle, les éléments d'une théorie de la prédication mise en place dans les Catégories : la distinction entre « être dit d'un sujet » (ut de subiecto dici) et «être dans un sujet» (ut in subiecto dici), introduite par Aristote au chapitre 2, en 1a20-21, avec ces mots : "Parmi les étants, les uns sont dits d'un sujet, mais ne sont en aucun sujet », - distinction qui fonde la distinction entre prédication univoque (attribution synonymique) et prédication accidentelle (attribution paronymique), parachevée par les médiévaux et tous ceux qui l'ont révisitée au fil des siècles en s'appuyant sur Avicenne.

\section{9 mars 2018}

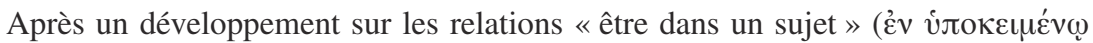

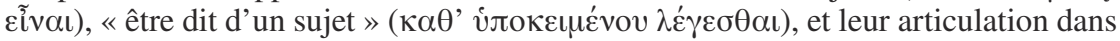
le «carré » d'Ammonius, on a repris le texte de 16b9-10 dont on a proposé la

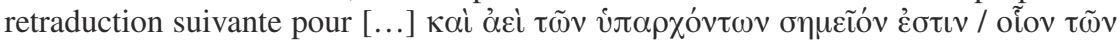
$\kappa \alpha \theta$ ' iлокеце́vov: [...] de plus il [le verbe] est toujours le signe de ce qui appartient / comme ce qui est dit d'un sujet. C'est cette formule : « ce qui appartient » (ici au pluriel), qui est reprise dans la suite immédiate (17a26-31) où Aristote propose une nouvelle combinatoire pour distinguer les quatre possibilités d'affirmation et de négation dans le cadre de la structure synthétique de l'apophansis comme ti kata tinos legein, i.e. les quatre possibilités de kataphasis (apophansis tinos kata tinos) et d'apophasis (apophansis tinos apo tinos). Ici on a souligné un nouveau problème de traduction. Tricot traduit : «Et puisqu'il est possible d'énoncer

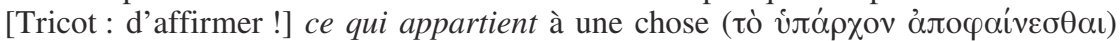

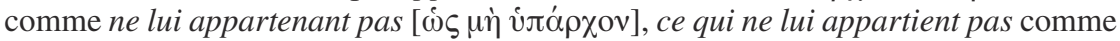
lui appartenant, ce qui lui appartient comme lui appartenant, ce qui ne lui appartient pas comme ne lui appartenant pas, et qu'on le peut également suivant les temps qui se trouvent en dehors du moment présent, tout ce qu'on a affirmé il sera possible de le nier, et tout ce qu'on a nié de l'affirmer. » Contrairement à Tricot, Boèce traduit

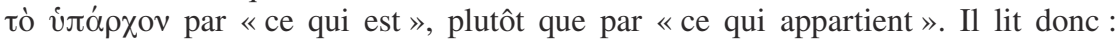
«Quoniam autem est enuntiare et quod est non esse et quod non est esse et quod est esse et quod non est non esse, etc. ». En fait, les deux traductions sont possibles. Il y a un double sens fondateur, "historial» (faisant histoire) du grec írá $\rho \chi 0 v$ : appartenance et existence. On en a donné une série d'exemples tirés du corpus des Ancient Commentators on Aristotle. Encouragés par ces résultats, on s'est intéressé à d'autres cas de mise en relation du Perihermeneias avec le reste du corpus aristotélicien. Pour ce faire, on est parti d'un passage de l'Expositio de 17a2-7 par Thomas d'Aquin sur le lieu de la vérité, qui connecte explicitement le Perihermeneias à la Métaphysique et aux Catégories : "Dicitur autem in enunciatione esse verum vel falsum sicut in signo intellectus veri vel falsi; set sicut in subiecto est verum vel falsum in mente, ut dicitur in VI Metaphisice, in re autem sicut in causa, quia, ut dicitur in libro Predicamentorum, eo quod res est vel non est, dicitur oratio vera vel falsa. » La réponse de Thomas superpose deux triangles : 1) celui constitué par les MOTS ou voix, ici : l'énonciation, autrement dit le discours extérieur, oral ; les CONCEPTS, ici l'esprit, la mens ; et les CHOSES, 2) celui du SIGNE, du SUJET, et de la CAUSE. L'articulation des deux triangles permet de poser trois locations : a) le vrai et le faux sont dans l'énonciation comme dans le SIGNE de l'intellection [pensée, intellect] du vrai et du faux ; b) dans l'esprit, comme dans un SUJET c) dans la chose comme dans leur CAUSE. Â ces trois locations correspondent trois modes d'être: 
a) sémantiquement ; b) subjectivement ; c) causalement. Pour ces deux derniers modes - subjectif et causal - qui ne sont pas dans le skopos du Perihermeneias, Thomas renvoie à la Métaphysique et aux Catégories.

Les deux références de Thomas montrent qu'une exégèse se construit en réseau selon une tradition interprétative, une histoire - traditionis traditio - qu'il appartient à l'archéologue et à l'historien de reconstruire et de questionner, non seulement dans sa trajectoire, mais aussi dans son ou ses aboutissements successifs, comme c'est le cas ici. La référence au livre VI de la Métaphysique s'entend de E 4, 1027b25-27, qui situe le lieu de la vérité dans la «PENSÉE » ( «Le faux et le vrai, en effet, ne sont pas dans les choses,... mais dans la pensée, ... la liaison et la séparation sont dans la pensée, et non dans les choses,... et « [ la cause] de l'Être en tant que vrai n'est qu'une affection de la pensée »), texte qui s'ajoute à $\Gamma 7,1011 \mathrm{~b} 26$, évoqué le 12 février 2018, qui situait la vérité au niveau du «DIRE » et à $\Theta 10,1051 \mathrm{~b}, 7-10$, qui, pour beaucoup d'interprètes, la situe au niveau des « CHOSES ». Ce trio de textes n'est pas le fait du hasard: il nous a été imposé à des moments divers par Foucault, Bouveresse, Heidegger et Thomas d'Aquin. Ce qu'il faut noter, c'est que l'on peut faire correspondre à chaque sommet du triangle thomasien (a, b, c : énonciation, esprit, chose) un passage différent de la Métaphysique (respectivement : $\Gamma$ 7, E 4, $\Theta 10$ ). La divergence des réponses d'Aristote aux questions les plus fondamentales de sa propre pensée est la donnée de base de l'histoire de la philosophie enclenchée par ce que Foucault appelle l'opération apophantique. Le triangle a, b, c articule ce que le sens commun et le langage ordinaire répartissent sur la différence entre vérité et réalité,

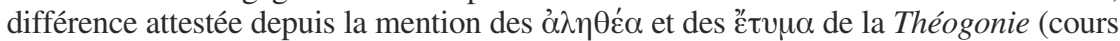
du 5 février). Le vrai se distingue du réel comme a,b de c : la vérité étant supposée être une caractéristique de ce que l'on dit $(=\mathrm{a})$ et de ce que l'on pense $(=\mathrm{b})$; la réalité, une caractéristique de ce qui est $(=\mathrm{c})$. Après une analyse du commentaire ammonien de 16a3-9 qui introduit expressément le triangle des phônai, noemata, pragmata, situe le lieu du vrai et du faux dans les pensées et, partant, dans les voix, mais " pas dans les choses prises en elles-mêmes, même composées ", on s'est intéressé à la seconde référence de Thomas, ouvrant sur un thème capital dans l'histoire de la vérité, l'histoire de l'Être et notre propre tentative de déconstruction du grand récit heideggérien : l'idée d'une vérité antéprédicative, d'une vérité dans les choses fondant ou plutôt causant la vérité/fausseté de l'énoncé déclaratif. On a montré que la route des choses partiellement fermée (ici) par Ammonius était rouverte par Thomas à l'aide de Cat. 4b8-10 : ce pourquoi un énoncé est dit vrai ou faux c'est l'être ou le non-

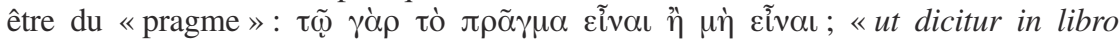
Predicamentorum, eo quod res est vel non est, dicitur oratio vera vel falsa ». On a suivi la fortune médiévale de cette formule, en rappelant l'ambiguïté non seulement du latin res, mais du grec $\pi \rho \tilde{\alpha} \gamma \mu \alpha$, partagés entre chose (individuelle) et état de choses (factuel), qu'il faut préserver sans doute jusque dans les Syncategoremata de Pierre d'Espagne, assurant que : «Res est causa veritatis orationis». On a conclu par quelques remarques sur les entités susceptibles d'être considérées aujourd'hui comme des vérifacteurs : états de choses (State of affairs), « tropes » (particuliers abstraits) et individus, en précisant que les éléments du trio vérité-réalité-existence s'articulaient dans le Truth-maker Principle («Principe du Vérifacteur»), dont la version standard, présentée et mise en œuvre par D.M. Armstrong est que «pour toute vérité contingente, voire pour toute vérité, qu'elle soit nécessaire ou contingente, il doit y avoir dans le monde quelque chose qui la rend vraie ». 


\section{6 mars 2018}

Répondant à une demande du public, on a «tracé » les notions de «valeur de vérité » et d' « état de chose », en présentant A) la distinction des quatre modes de l'effectivité (Wirklichkeit) chez Herman Lotze (1817-1881): l'être (Sein) des choses; l'advenir (Geschehen) des événements ; la subsistance ou consistance (Bestehen) des rapports (mathématiques); la validité (Geltung) des propositions (logiques), puis B) l'introduction de la Wahrheitswert par Wilhelm Windelband (1848-1915), qui coordonne la notion de valeur - Wert - et celle de validité - Geltung - et qui, en tant que (néo-)kantien, s'oppose au relativisme nietzschéen et à l'interprétation nietzschéenne de la volonté de vérité : pour lui, la volonté n'engendre ni ne produit la vérité (où l'on rejoint la critique de Foucault par Bouveresse). On a ensuite examiné archéologiquement quelques-unes des grandes théories médiévales des vérifacteurs, laissées de côté aussi bien par Heidegger que par Foucault. On a d'abord présenté la théorie des trois types de composition : réelle, mentale, phrastique, dans le sophisma «Omnis homo de necessitate est animal» (noté ici OHNEA) du philosophe danois Boèce de Dacie († avant 1284), la composition réelle causant la vérité de la composition mentale, et celle-ci la vérité de l'énoncé oral. De là, on est passé à l'examen de la théorie des trois sortes de propositions - réelles, mentales et orales - soutenue par Gauthier Burley dans son Exposition sur le Perihermeneias de 1337. Selon Burley, dans la formule « ex eo quod res est vel non est propositio (oratio) dicitur esse vera vel falsa » de Cat. 8b4-10 et $14 \mathrm{~b} 21-22$, res a le sens de res significata per totam propositionem: la réalité signifiée par la proposition (mentale/orale) tout entière. Cette «res qui est ou n'est pas » est une proposition constituée de choses, une réalité complexe qui fonde la « vérité complexe », i.e. la composition et la division « intellectuelles ». Les théories de Boèce de Dacie ou de Gauthier Burley étant caractéristiques d'un type de problèmes abordé dans la littérature et la pratique académiques de ce qu'on appelle à partir du XIII ${ }^{\mathrm{e}}$ siècle les sophismata, c'est tout un pan de l'histoire de la pérennisation du sophisme, accompagnant l'exclusion du sophiste selon Foucault, qui est venu au jour. On en a évoqué quelques figures, en faisant remarquer que les deux premiers auteurs de sophismata OHNEA mentionnés par un historien francophone (Pierre Mandonnet, en 1899), Siger de Brabant et Boèce de Dacie, étaient aussi associés dans l'historiographie à la condamnation de l'averrö̈sme parisien en 1277. On a suggéré que l'histoire des sophismata croisant celle des condamnations, et la quête de la vérité celle du pouvoir, le Magistère (le Pape, les évêques, les structures de pouvoir internes aux ordres religieux) prenait fonctionnellement au Moyen Âge la place des Maîtres de vérité dans le dispositif de la Grèce archaïque. On a pour illustrer ce point évoqué la condamnation à Oxford, le 18 mars 1277, par Robert Kilwardby, évêque de Cantorbéry, chancelier d'Oxford, d'une thèse de logique modale subordonnant la vérité d'une proposition modale nécessaire à l'existence du référent désigné par le terme sujet de la proposition (la même thèse étant soutenue par certains, comme le franciscain Roger Bacon pour les propositions de inesse correspondantes, universelles comme Tout homme est un animal, ou particulières comme César est un homme). On a examiné quelques théories syntacticosémantiques du XIII ${ }^{\mathrm{e}}$ siècle destinées à éviter de s'engager sur la voie du «troisième domaine », puis passant au XIV $\mathrm{X}^{\mathrm{e}}$, on a montré que celui-ci avait vu émerger un problème nouveau, favorisé par l'introduction d'un concept nouveau - celui de «signifiable complexement » ou de «signifiable par complexe», le significabile 
complexe (noté ici SC) - centré non plus sur la référence vide, mais sur l'existence de vérités antérieures à l'existence du monde, comme précisément (le) mundum fore, le fait que le monde serait. En s'appuyant sur l'exégèse de Mc 14, 30 par Grégoire de Rimini, axée sur la question de savoir si «Petrum esse peccaturum in $A$ » fut vrai de toute éternité, on a exposé en détail la théorie de la "dénomination extrinsèque » fondant la solution que nous avons appelée du « Grand Juge », qui fait originairement de la modalité aléthique «vrai » un attribut du Juge (Dieu) et de son acte de juger éternel, non de l'objet du jugement ou de son contenu, qui sont dits «vrais » par dénomination extrinsèque à partir de leur cause. On a montré que cette théorie retrouvait le modèle de la double «conformité » ou adéquation (rei ad intellectus, intellectus ad rem) décrite par Heidegger (analysée dans le cours du 20 mars 2017). Enfin on a fait remarquer que les débats sur le signifiable par complexe s'inscrivaient dans une longue histoire, qui avait vu s'opposer du Moyen Âge à nos jours ceux qui soutenaient qu'il y a des vérités indépendantes de tout intellect, de toute subjectivité pensante, y compris divine (hypothèse du «nemine cogitante») et ceux qui maintenaient que toute vérité a besoin d'un porteur - Dieu en dernière instance, à titre d'archi-sujet transcendantal.

Faute de temps, il n'a pas été possible de présenter en détail les conclusions du cours. On a dû se limiter à deux conclusions générales : C1 : l'argument d'une réduction foucaldienne de la vérité au tenir-pour-vrai s'inscrit dans un débat aussi vieux que l'interprétation de la théorie aristotélicienne de la vérité ; C2 : il ne faut pas reprocher à Foucault d'avoir réduit l'un à l'autre. Il faut plutôt déplorer qu'il n'ait pas poussé plus avant l'analyse, et qu'il ait finalement exclu Aristote, et avec Aristote, le Moyen Âge, d'un récit plus détaillé de la «pérennisation du sophisme » complétant celui de l'exclusion du sophiste.

On y a ajouté quelques conclusions et remarques particulières soit oralement, soit dans les diapositives du « support du cours » (accessible sur le site du Collège de France). C3 : Concernant le dossier du "philosophe essentiel », on a indiqué une rupture avec Heidegger allant au-delà des éléments évoqués par D. Defert dans la situation du cours sur le Courage de la vérité. Il s'agit d'un passage du manuscrit du cours du 29 février 1984, non prononcé par Foucault, où celui-ci soutient qu' « il faut perdre l'habitude de [...] penser [...] le nihilisme [...] sous la forme d'un destin propre à la métaphysique occidentale, destin auquel on ne pourrait échapper qu'en revenant à ce dont l'oubli a rendu possible cette métaphysique elle-même ». Cette prise de position sur le nihilisme - qui met expressément en question la lecture heideggérienne de Nietzsche - est solidaire d'une proposition d'ensemble exprimée par la notion de " manière de vivre », et l'attention portée au cynisme - un des sujets majeurs du cours de 1984 - qui sont liées à une certaine intégration de vues de Pierre Hadot sur l'espèce de coupure épistémologique instaurée entre philosophie antique, d'une part, christianisme, pensée médiévale et scolastique, d'autre part (cf. p. 175 : «La combinaison du cynisme et du du scepticisme au XIX ${ }^{e}$ siècle a été au principe du nihilisme entendu comme manière de vivre dans une certaine attitude à l'égard de la vérité »). C4 : concernant le rapport de Foucault au Moyen Âge on a proposé un schéma représentant les deux regroupements des modalités alèthurgiques qui caractérisent la période médiévale dans Le Courage de la vérité (p. 28-29): 1) regroupement de la modalité prophétique et de la modalité parrêsiastique avec les "grands prédicateurs »; 2) regroupement de la modalité de la sagesse et de la modalité de l'enseignement avec «l'Université ». On a brièvement discuté la thèse de Foucault faisant de la «Prédication» et de «l'Université » des «institutions 
propres au Moyen Âge, dans lesquelles on voit se regrouper, deux par deux », les quatre «grands modes de véridiction», regroupement qui définit «un régime de véridiction, un régime du dire-vrai très différent de celui qu'on pouvait trouver dans le mode hellénistique et gréco-romain », qui combinait plutôt «parrêsia et sagesse ». On a en quelques mots souligné l'importance de la partie conclusive du cours du 28 mars 1984, non prononcée (conservée dans le manuscrit du cours et éditée p. 309311 du Courage de la vérité par F. Gros). «Il vaudrait mieux présenter les choses ainsi »: c'est la véritable articulation du travail de Foucault sur la philosophie antique qui s'énonce ici ; c'est elle qui fournit un contre-récit au récit heideggérien d'une histoire de l'être axée sur l'oubli de l'être et les deux commencements ( Premier» et «Nouveau commencement»), mais aussi sur le couple PlatonAristote placé par Foucault lui-même dans les $L V S$ au point de départ de l'opération apophantique ; contre-récit qui éclaire rétrospectivement l'élimination d'Aristote. C'est sur un autre couple que le couple Platon-Aristote des LVS que se fonde Foucault en 1984 : celui que forment Platon et le cynisme. La thèse centrale est que «la philosophie ancienne a lié l'un à l'autre le principe du souci de soi (devoir de s'occuper de soi-même) et l'exigence du courage de dire, de manifester la vérité », en naviguant entre les deux modalités opposées constituant l'héritage de Socrate : la modalité platonicienne et la modalité cynique.

Quelques critiques: 1) Foucault reconduit l'éloge de la «manière de vivre» comme spécificité de la philosophie antique et le discrédit relatif du christianisme médiéval dans le schéma philosophique et historiographique de P. Hadot, marqué notamment par la dissociation entre théologie scolastique et vie spirituelle, qui projette sur le monde universitaire une certaine vision de la différence entre l'école et le cloitre dans les siècles pré-universitaires. 2) On doit réviser cette vision de l'Université, qui exclut l'êthos académique, dissocie sagesse et parrêsia dans l'institution, et qui, surtout, échouant à trouver au Moyen Âge un «dire-vrai philosophique » reproduisant à l'identique le schéma «antique» ne voit pas la réalité, la portée et la signification philosophiques du dire-vrai du théologien ou du juriste de l'âge scolastique.

Quelques propositions : 1) Concernant l'Université : il faut reconnaître le statut de la disputatio, au-delà de la simple tekhnê, comme «forme de vie» et modalité originale du «dire-vrai » philosophique. 2) Concernant la Prédication: il faut prendre le mesure du rôle des « prédicateurs » dans la cura animarum. La prédication de Meister Eckhart relie les quatre sommets du quadrangle foucaldien (SAGESSE, être ; ENSEIGNEMENT, tekhnê ; PROPHÉTIE, destin ; PARRÊSIA, êthos), au croisement des deux diagonales [prophétie-parrêsia $=$ Prédication $;$ sagesse-enseignement $=$ Université], qui marque la place d'une autre modalité originale, proprement médiévale, du «dire-vrai» philosophique, articulant/opposant autrement le «platonisme» (contemplation, souci de soi) et le «cynisme» (exercice, ascèse, courage de la vérité) distingués par Foucault. Le Lesemeister est Lebemeister: le maître à lire est maître à vivre. 3) Il faut revenir sur le bios theoretikos évacué par Foucault en même temps que le désir naturel de savoir : ce « bonheur théorique » qui est la finalité même de l'existence humaine - voire de la société humaine : la « félicité mentale », reprise chez Dante, la fiducia philosophantium péripatéticienne, averroïste, bref l'articulation gréco-arabo-latine de la philosophie de l'esprit, de l'éthique et de la métaphysique. Enfin, il faut revisiter la théologie médiévale - ce que l'on a essayé de faire depuis 2014 et continuera en 2019, pour finir. 
COLLOQUE - L'EUROPE DE LA LOGIQUE. LES TRADITIONS ARISTOTÉLICIENNES MÉDIÉVALES ET MODERNES EN CONTEXTES : EMPIRES ET POLITIQUES DE LA LOGIQUE

Le séminaire a pris la forme d'un colloque international organisé en collaboration avec Julie Brumberg-Chaumont (CNRS/LEM/PSL) et Antonella Romano (EHESS/ Centre Alexandre Koyré), dans le cadre du projet PSL (CNRS/EPHE/Collège de France/EHESS) : «L'Europe de la logique. Les traditions aristotéliciennes médiévales et modernes en contextes : empires et politiques de la logique » (dir. J. Brumberg-Chaumont), les 14 et 15 mai 2018 au Collège de France.

À rebours d'une pratique historiographique exclusivement occupée à la restitution et à l'évaluation des doctrines logiques passées, le colloque visait à jeter les bases d'une recherche collective prenant acte des dimensions institutionnelles, territoriales, confessionnelles, politiques, éducatives et anthropologiques revêtue par l'histoire des traditions logiques aristotéliciennes dans l'Europe médiévale, renaissante et moderne. En pluralisant l'espace européen pour l'étendre à ses missions et à ses colonies ainsi qu'à ses composantes byzantines, musulmanes et juives, tout en insérant l'histoire de la logique dans l'orbite du renouveau méthodologique de l'histoire des sciences et de la philosophie, on s'est appuyé sur l'histoire des pratiques logiques, en particulier éducatives, pour dénaturaliser et historiciser la notion de «logique». Dans sa communication intitulée "Le split Oxford/Paris », Alain de Libera a dressé le bilan de cinquante ans de recherche sur les traditions universitaires parisienne et oxonienne de l'enseignement de la logique au XIII' siècle.

Intervenants : Julie Brumberg-Chaumont (PSL/CNRS/LEM), Alain de Libera (Collège de France), Andrew Laird (Brown University, États-Unis), Marco Sgarbi (Università Ca' Foscari, Venezia), Christophe Geudens (KU Leuven), Steven Coesemans (KU Leuven), Jacob Schmutz (Sorbonne Université, Paris), David Simonetta (Collège de France), Catherine König-Pralong (université de Fribourg/ EHESS), Christophe Erismann (université de Vienne), Daniel de Smet (PSL/CNRS/ LEM), Charles Manekin (University of Maryland, États-Unis), John Marenbon (Trinity College, Cambridge), Costantino Marmo (université de Bologne), Irène Rosier-Catach (PSL/EPHE/CNRS/HTL).

\section{RECHERCHE}

\section{Groupe Actualité médiévale (GAM). JouRnÉES D’ÉTUdeS}

Pour appréhender la place du Moyen Âge dans l'histoire de la philosophie et penser son lien avec la modernité, le GAM, animé par Ángela Beatriz Ávalos-Soto (ATER, Collège de France), et la chaire Histoire de la philosophie médiévale ont organisé les 30 et 31 mai 2018 deux journées d'études consacrées aux figures paradigmatiques d'Étienne Gilson, 1884-1978 (sous la direction de Alain de Libera et Florian Michel, Paris 1 Panthéon-Sorbonne), et de Hans Blumenberg, 1920-1996 (sous la direction de Aurélien Robert, CNRS, et Christophe Grellard, EPHE). Dans sa communication intitulée «Translatio studiorum : retours sur un héritage gilsonien », Alain de Libera a évoqué dans leur continuité les contributions respectives d'Étienne Gilson, de Jean Jolivet et de Marc Geoffroy (tous deux disparus en 2018) à l'histoire des réceptions médiévales, latine et juive, de la philosophie arabo-musulmane. 
Intervenants :

Journée Gilson (30 mai 2018): Alain de Libera, Florian Michel, ThierryDominique Humbrecht, O.P. (Institut catholique de Toulouse), Michel Fourcade (université de Montpellier), Ruedi Imbach (Sorbonne Université, université de Fribourg).

Journée Blumenberg (31 mai 2018): Christophe Grellard, Aurélien Robert, Olivier Boulnois (EPHE, Ve section, LEM), Joël Biard (université de Tours, CSER), Philippe Büttgen (Université Paris 1 Panthéon Sorbonne); Jean-Claude Monod (CNRS, UMR 8547, Archives Husserl).

\section{Publications}

LIBERA A. de, «Logique et anthropologie : averroïsme et platonisme selon Buridan et Nifo », in C. GRellard (dir.), Miroir de l'amitié: mélanges offerts à Joël Biard, Paris, Vrin, coll. «Études de philosophie médiévale », nº 106, 2017, p. 329-352.

LiberA A. de, « Les Latins parlent aux Latins », in P. BÜTTGEN, A. de LiBERA, M. RASHED et I. ROSIER-CATACH (dir.), Les Grecs, les Arabes et nous : enquête sur l'islamophobie savante, Le Caire, El Gebalaya St. Opera House, El Gezira, 2017, p. 201-245 [traduction arabe de Paris, Fayard, coll. «Ouvertures », 2009, p. 175-215].

Libera A. de, La Volonté et l'Action. Cours du Collège de France 2015, Paris, Vrin, coll. «Bibliothèque d'histoire de la philosophie », 2017.

LiBera A. de, « Le Centaure et le Schimmel. Onto-logique d'une fiction dans la Psychologie du point de vue empirique », in D. FISSETTE et G. FrÉCHETTE (dir.), « Franz Brentano », Revue philosophique de la France et de l'étranger, tome 142, no 4, 2017, p. 471-489.

LIBERA A. de, «Présentation et représentation. Aux origines du "représentationnalisme" », in J. Pelletier et M. Roques (dir.), The Language of Thought in Late Medieval Philosophy. Essays in Honor of Claude Panaccio, Cham, Springer, coll. « Historical-Analytical Studies on Nature, Mind and Action », n 5 , 2018, p. 417-435.

LiBERA A. de, «Entre Tolède et Hamadan. La Logica d'Avicenne », Introduction doctrinale à Avicenne. Logica (Logique du Šifá), texte latin, édition critique de la traduction médiévale par F. HUdRY, Paris, Vrin, coll. « Sic et Non», 2018, p. 9-66.

LiberA A. de, «Un peu de Crémant dans l'eau froide », Critique, vol. 853-854, nº 6, 2018, p. 628-638.

LIBERA A. de, « Nihilisme - Nonisme - Néminisme : De Goetz à Stirner », in T. IREMADZE et U.R. JECK (dir.), Veritas et subtilitas : Truth and Subtlety in the History of Philosophy. Essays in memory of Burkhard Mojsisch 1944-2015, Amsterdam, John Benjamins Publishing Company, coll. « Bochumer Studien zur Philosophie», nº 59, 2018, p. 349-379. 
